\title{
Spin Switching via Quantum Dot Spin Valves
}

\author{
N. M. Gergs, ${ }^{1}$ S. A. Bender, ${ }^{1}$ R. A. Duine,,${ }^{1,2}$ and D. Schuricht ${ }^{1}$ \\ ${ }^{1}$ Institute for Theoretical Physics, Center for Extreme Matter and Emergent Phenomena, Utrecht University, \\ Leuvenlaan 4, 3584 CE Utrecht, The Netherlands \\ ${ }^{2}$ Department of Applied Physics, Eindhoven University of Technology, $5600 \mathrm{MB}$ Eindhoven, The Netherlands
}

(Received 28 June 2017; revised manuscript received 13 September 2017; published 5 January 2018)

\begin{abstract}
We develop a theory for spin transport and magnetization dynamics in a quantum dot spin valve, i.e., two magnetic reservoirs coupled to a quantum dot. Our theory is able to take into account effects of strong correlations. We demonstrate that, as a result of these strong correlations, the dot gate voltage enables control over the current-induced torques on the magnets and, in particular, enables voltage-controlled magnetic switching. The electrical resistance of the structure can be used to read out the magnetic state. Our model may be realized by a number of experimental systems, including magnetic scanning-tunneling microscope tips and artificial quantum dot systems.
\end{abstract}

DOI: 10.1103/PhysRevLett.120.017701

Introduction.-The reliable manipulation and detection of magnetic moments by electrical means remains one of the overarching themes of spintronics. Recent years have seen the development of several techniques involving a variety of materials (conducting, insulating, and semiconducting) and heterostructures to this end. A key observation is that the total conductance of metallic magnetic multilayers may be extremely sensitive to the magnetic orientations of the constituent magnets [1-4], owing to the spin-dependent transport coefficients of the various components; the "giant magnetoresistance" of such heterostructures demonstrates the possibility of electrically reading the magnetic state of microlayers and has been employed in mass produced devices shortly thereafter. Subsequently, it was shown that the generation of magnetic dynamics leads to switching of magnetic multilayers by large electrical currents, which become spin polarized and thus transfer spin across the structure [5-10]. Typically, components of such heterostructures are sufficiently large that interactions and quantum effects do not play a prominent role in transport. As devices are scaled down, however, these effects become increasingly significant. Quantum dots coupled to ferromagnetic leads, which can be viewed as a nanoscale analogue to magnetic multilayer spin valves, represent an extreme scaling down of the metallic interlayer. These quantum dot spin valves have proven a fertile subject of research on spin-dependent quantum transport in recent years [11-19]. In these studies, the ferromagnetic leads are static reservoirs of angular momentum; if, however, the reservoir magnetic moments are sufficiently small and the electric currents sufficiently large, the reservoir moments may be reoriented by the absorption of the spin current (i.e., spin-transfer torque), just as in magnetic multilayers. In contrast to multilayers, however, wherein spin-transfer torques are controlled by the source-drain bias alone, in ferromagnet-dot-ferromagnet tunnel junctions, gating of the dot provides a new route of electrical manipulation of magnetic dynamics, opening up rich new phase behavior for the magnetic orientations of the ferromagnetic reservoirs.

In this Letter, we discuss how transport through a spindegenerate quantum dot can be utilized to manipulate attached nanomagnets via applied gate and bias voltages. These voltages control the electronic transport through the quantum dot, which, in turn, induces spin torques in the nanomagnets. Since the electronic transport can be well controlled by the gate and bias voltage, the spin torques can be tuned as well, which is not straightforwardly possible in standard spintronics setups like magnetic multilayers. We demonstrate that the tunablility of the spin torques enables the magnetic switching of the nanomagnets between parallel and antiparallel configurations, which are experimentally distinguishable by their magnetoresistance and thus can be readout electrically. Moreover, we find a new resonance for nearly parallelly aligned reservoirs, which turns out to be a partner resonance to the recently reported [19] spin resonance in the absence of spin splitting.

We suppose a separation of time scales between "fast" quantum electron transport and "slow" magnetic dynamics of the reservoirs. While such an approach assumes low magnetic frequencies, and thus that charge- and spinpumping effects are negligible, it allows us to first treat electron transport for quasistatic, arbitrary magnetic orientations of the reservoirs. We then use the resulting expressions for the spin-polarized current that flows through the structure to obtain spin torques on the magnetic reservoirs, which drive magnetic dynamics. This Letter thus combines two approaches. The first is a semiclassical treatment of the spin torques and "slow" magnetic dynamics from within a Landau-Lifshitz-Gilbert (LLG) phenomenology. The second deals with the "fast" electron transport 
through the quantum dot or quasistatic magnetic reservoirs; the quantum dot spin valve transport properties we obtain are quite general and apply to a wide range of magnetic systems in which a quantum dot might be embedded.

Magnetic dynamics. - To model magnetic dynamics of the reservoirs, induced by electronic transport discussed below, we treat their respective magnetic moments as single-domain macrospins subject to the LLG equations modified to incorporate spin torques [20]

$$
S \frac{d \vec{n}_{r}}{d t}=-\mu_{0} \gamma S \vec{n}_{r} \times \vec{H}_{r}+\vec{I}_{S, r}^{\perp}-\alpha S \vec{n}_{r} \times \frac{d \vec{n}_{r}}{d t},
$$

where $S$ is the macrospin of the reservoirs, $r \in\{S, D\}$ denotes the source and drain with the macrospin orientations $\vec{n}_{r}\left(\left|\vec{n}_{r}\right|=1\right), \gamma$ is the absolute value of the gyromagnetic ratio, $\mu_{0}$ the vacuum permeability, and $\alpha$ is the phenomenological Gilbert damping. The effective magnetic field in the reservoirs is given by $\vec{H}_{r}=1 /\left(\mu_{0} \gamma S\right)$ $\left(\delta E_{r} / \delta \vec{n}_{r}\right)+\vec{H}_{\text {thermal }}$. We consider the simple case of an easy-axis energy $E_{r}=-(K V / 2)\left(\vec{n}_{r} \cdot \vec{e}_{\mathrm{z}}\right)^{2}$, which facilitates two degenerate magnetic equilibria at $n_{r}^{z}= \pm 1$. Motivated by materials like Galfenol $[21,22]$ (iron-gallium alloys), we have also analyzed [23] the case of cubic anisotropy and found that all qualitative features remain unchanged. Furthermore, $\vec{H}_{\text {thermal }}$ implements the influence of the temperature $T$ via fluctuations with Gaussian noise of variance [29] $\sigma_{\text {thermal }}^{2}=2 \alpha T /\left(\gamma^{2} \mu_{0}^{2} S\right)$. Finally, $\vec{I}_{S, r}^{\perp}$ is the component of the electronic spin current $\vec{I}_{S, r}$ impinging on the reservoirs that is perpendicular to the macrospin orientation $\vec{n}_{r}$. Whereas the parallel component is carried into the bulk of the reservoir by itinerant electrons, $\vec{I}_{S, r}^{\perp}$ is absorbed by reorienting $\vec{n}_{r}$ and hence, enters as a spin torque [30]; this current, which gives rise to magnetic dynamics and switching, is highly sensitive to interactions in the magnetic dot and will be calculated and discussed in detail in the next subsection. The spin current can be further decomposed [20] into an out-of-plane spin current $I_{\mathrm{FL}, r}$ that acts fieldlike in the LLG equation (1) and an in-plane dampinglike contribution $I_{\mathrm{DL}, r}$

$$
\vec{I}_{S, r}^{\perp}:=\left(\vec{n}_{r} \times \vec{e}_{\mathrm{z}}\right) I_{\mathrm{FL}, r}+\vec{n}_{r} \times\left(\vec{n}_{r} \times \vec{e}_{\mathrm{z}}\right) I_{\mathrm{DL}, r} .
$$

For simplicity, we will limit our discussion to the case where the magnetization direction of only the drain is able to rotate freely, with the source macrospin $\vec{n}_{S}$ fixed at $\vec{e}_{\mathrm{z}}$. For the case that both nanomagnets are able to rotate freely, no qualitative changes occur inside the Coulomb blockade regime where the quantum dot is singly occupied.

The perpendicular spin current $\vec{I}_{\mathrm{S}, \mathrm{r}}^{\perp}$ in Eq. (1) includes all effects of the coupling of the two nanomagnets via the quantum dot device. In the next section, we discuss the quantum dot, the electronic transport, and the resulting spin current $\vec{I}_{S, r}$.
Transport.-We consider as a simple model an Anderson impurity with a spin-degenerate energy level coupled to generally noncollinear magnetic reservoirs. All qualitative conclusions are quite general and expected to hold also for systems with, for example, multiple energy levels or weak to moderate electron-phonon coupling as none of these fundamentally change the equation of motion for the dot spin. The dot and tunneling Hamiltonians are given by $H_{\mathrm{dot}}=\sum_{\sigma} \varepsilon n_{\sigma}+U n_{\uparrow} n_{\downarrow}, n_{\sigma}=d_{\sigma}^{\dagger} d_{\sigma}, \sigma=\uparrow, \downarrow$, and $H_{\text {tun }}^{r}=\sum_{\sigma \sigma^{\prime} k} t_{r} d_{\sigma^{\prime}}^{\dagger} R_{\sigma^{\prime} \sigma}^{r} c_{\sigma k r}+$ H.c., $\quad r \in\{\mathrm{S}, \mathrm{D}\}, \quad$ with $d_{\sigma}$ and $c_{\sigma k r}$ denoting the electron annihilation operators on the dot and in the reservoirs, and $t_{r}$ is the corresponding tunnel coupling. A sketch of the setup is given in Fig. 1. In the following, we will use the notation $\tilde{\varepsilon}:=\varepsilon+U / 2$, which is the particle-hole-symmetrized [31] on-site energy. In both reservoirs, we consider a polarization implemented via the density of states $\rho^{\sigma}$ of spin $\sigma$; i.e., we set $p:=$ $\left(\rho^{\uparrow}-\rho^{\downarrow}\right) /\left(\rho^{\uparrow}+\rho^{\downarrow}\right)$ and $\rho_{0}=\left(\rho^{\uparrow}+\rho^{\downarrow}\right) / 2$. Furthermore, $R^{r}$ is a matrix that encodes the different polarization direction of the reservoirs. Without loss of generality, we choose for the source $R_{\sigma \sigma^{\prime}}^{D}=\delta_{\sigma \sigma^{\prime}}$, and hence, for an opening angle $\theta$ between both reservoir polarization directions, we obtain $R_{\sigma \sigma^{\prime}}^{D}=\delta_{\sigma \sigma^{\prime}} \cos (\theta / 2)+\sigma\left(1-\delta_{\sigma \sigma^{\prime}}\right) \sin (\theta / 2)$. The transport properties of this model have already been studied $[12,14,19,32,33]$ in some detail. In contrast, here, we focus on the spin torques and, in particular, their consequences for the dynamics of the reservoir nanomagnets.

To compute the transport through this strongly correlated quantum dot, we treat dot-reservoir tunnel coupling perturbatively [23,31,34-38]. The corresponding perturbative scale is given by $\Gamma=\frac{1}{2} \sum_{r} \Gamma_{r}$, with $\Gamma_{r}:=2 \pi t_{r}^{2} \rho_{0}$; i.e., we assume $\Gamma \ll T$. All simulations are carried out with a step size of $\Delta t=10^{-2} \Gamma$. We assume a flat density of states for the reservoirs with an energy cutoff $D$ much larger than all other energy scales in the model. We include all $\mathcal{O}(\Gamma)$ and $\mathcal{O}\left(\Gamma^{2}\right)$ effects like, e.g., cotunneling in a consistent way regarding the occurring relaxation rates of the density operator. From this, we determine the stationary transport properties of the model. The underlying assumption behind using the stationary values is that the time scale for the dot-relaxation processes to happen is much smaller than the

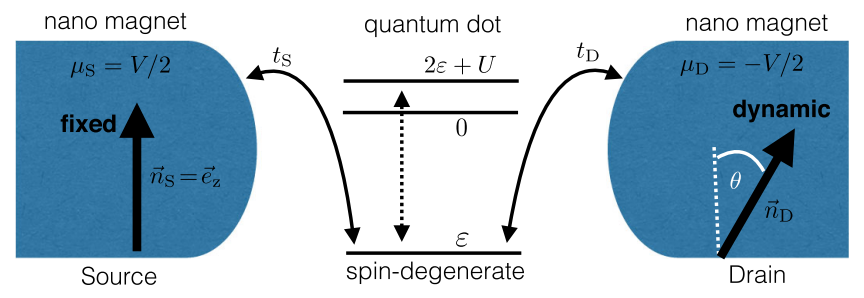

FIG. 1. Sketch of the system: A spin degenerate single-level quantum dot is coupled via tunnel couplings $t_{S / D}$ to ferromagnetic source and drain reservoirs, which are held at different chemical potentials $\mu_{S / D}= \pm V / 2$. The orientation $\vec{n}_{S}$ of the source reservoir is fixed while the macrospin $\vec{n}_{D}$ of the drain can fluctuate. 
time scale for the resulting dynamics of the nanomagnets described by (1).

Albeit we consider a simple model for the dot Hamiltonian, the non-spin-conserving tunnel couplings to the reservoirs yield a variety of spin phenomena to explore. In the equations of motion describing the dot dynamics, the finite reservoir polarizations yield an induced magnetic field on the dot given by [14]

$$
\vec{B}_{\text {ind }}=\frac{1}{\pi} \sum_{\substack{r=S, D \\ q= \pm}} \Gamma_{r} \vec{n}_{r} p_{r} q \operatorname{Re} \psi\left(\frac{1}{2}+i \frac{\tilde{\varepsilon}-\mu_{r}-q U / 2}{2 \pi T_{r}}\right),
$$

with $\psi$ denoting the digamma function. The induced effective field (3) is included in the nonvanishing leading order [23]. We note that the near degeneracy of the dot level is crucial as a large Zeeman splitting would pin the dot polarization direction and suppress the effects associated to the induced magnetic field. Such a degeneracy can also be realized in the presence of strong external magnetic fields by, for example, fine tuning [39] different valley degrees of freedom.

Spin resonance condition.-Crucial for the understanding of our results is the fact that the degenerate spin level of the quantum dot leads, in combination with the induced effective magnetic field (3) from the reservoirs, to a nontrivial spin resonance [19]. The resonance condition relevant for the dynamics of the nanomagnets is given by (generalizing the result of Ref. [19])

$$
\vec{B}_{\text {ind }} \cdot\left(\vec{n}_{D}-\vec{n}_{S}\right)=0,
$$

which is the condition for the effective magnetic field (3) to change the dot spin from one initial reservoir polarization direction to the other with maximal efficiency, i.e., fastest in time and with the smallest necessary thermal fluctuations. The resonance condition (4) results in a relation between the bias voltage $V$ and on-site energy $\tilde{\varepsilon}$, which is independent of the opening angle, provided that $\theta \neq 0$. For a symmetric setup $p_{S} \Gamma_{D}=p_{D} \Gamma_{S}$, the resonance condition reduces to $V=0$ for which transport is suppressed [19].

Switching diagram.-We will now turn to our main result and discuss the spin dynamics. For already small deviations from the parallel alignment $\theta \gtrsim 0.1 \pi$, the fieldlike spin torque dominates over the dampinglike contribution; see Eq. (2) for the definitions. Depending on the sign of the fieldlike torque, the driven magnetic ground state will be either a parallel or an antiparallel alignment of the nanomagnets; we refer to these as the parallel and antiparallel phases, respectively.

In Fig. 2, we show the corresponding phase diagram (see also [23]). The plotted average opening angle between the source and drain reservoirs is obtained as follows: the system is initialized in a parallel configuration $\theta_{\text {init }}=0$ (recall that the orientation of the source spin is held fixed). The finite temperature encoded in the variance $\sigma_{\text {thermal }}$ leads to fluctuations in $\theta$ which are, depending on the point in the $(\tilde{\varepsilon}, V)$-phase diagram, suppressed or enhanced by the spin

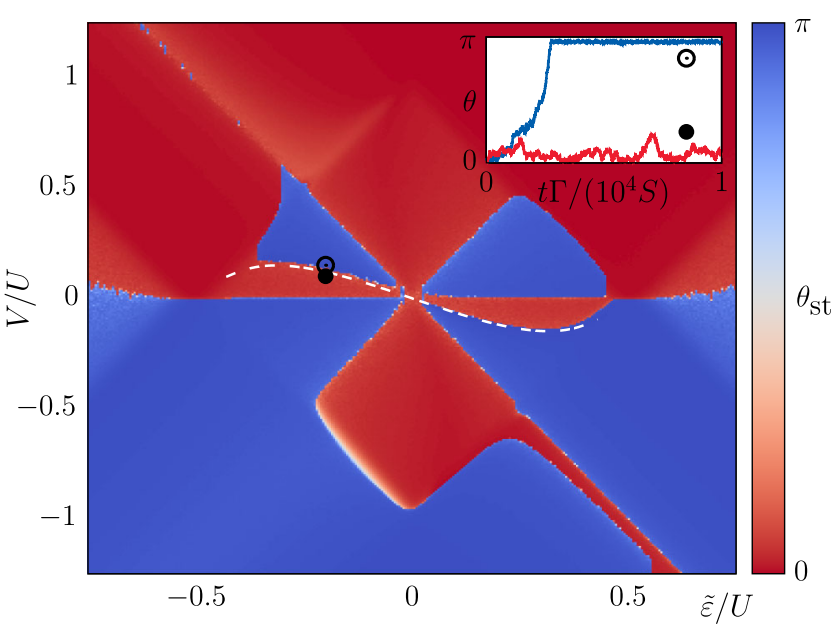

FIG. 2. Switching diagram displaying the average opening angle between the reservoirs (see text for the precise definition). The parameters are given by $\Gamma_{D}=2 \Gamma_{S}, U=10^{2} \mathrm{~T}=10^{3} \Gamma$, $p=0.99, K V=0.01 \Gamma, \alpha=0.1$, and $\sigma_{\text {thermal }}^{2}=10^{-4}$. The resonance (4) is indicated by the white dashed line. Inset: Two exemplary time evolutions of the opening angle for the parameters $\tilde{\varepsilon}=0.2 U$ and $V=0.1 U$ as well as $V=0.175 U$.

torque acting on $\vec{n}_{D}$; two exemplary time evolutions of the angle $\theta$ are shown in the inset. (We note that the macrospin length $S$ only rescales the time axis.) The average opening angle is obtained by performing the time evolution up to $t=$ $10^{4} \mathrm{~S} / \Gamma$ and then averaging $\theta$ from there to $t=2 \times 10^{4} \mathrm{~S} / \Gamma$. In the phase diagram, we see that the average opening angle indeed ends up in either a parallel or an antiparallel configuration and that this can be controlled by the values of the gate and bias voltages $\varepsilon=\tilde{\varepsilon}-U / 2$ and $V$, respectively. For example, the resonance (4) indicated by the white dashed line corresponds to a phase boundary. The precise value of the stationary angle depends on the parameters in particular and also on the temperature. We note that the lack of symmetry of the switching diagram under $(\tilde{\varepsilon}, V) \rightarrow(-\tilde{\varepsilon},-V)$ is due to the fixing of the source magnetization $\vec{n}_{S}$.

A transition between parallel and antiparallel phases can be easily detected by measuring the charge transport through the device. Let us compare three cases: one in which the drain magnetization is fixed in the parallel configuration $(\theta \approx 0)$, one in which it is fixed in the antiparallel configuration $(\theta \approx \pi)$, and one in which the relative orientation $\theta_{\text {st }}$ is determined by the applied voltages as shown in the phase diagram Fig. 2. As shown in Fig. 3, charge transport is qualitatively distinct between the three cases. For example, the resonance (4) does not [19] cause a significant feature in the parallel case, while in the other two cases, it is clearly visible in the charge transport. The insets in Fig. 3 show the fieldlike (red lines) and dampinglike (blue lines) spin torques along cuts at $\tilde{\varepsilon}=-0.3 U$, revealing nontrivial dependencies on the applied gate and bias voltages. In particular, we conclude that the quantum dot setup allows control over the relative strength of the spin torques. 


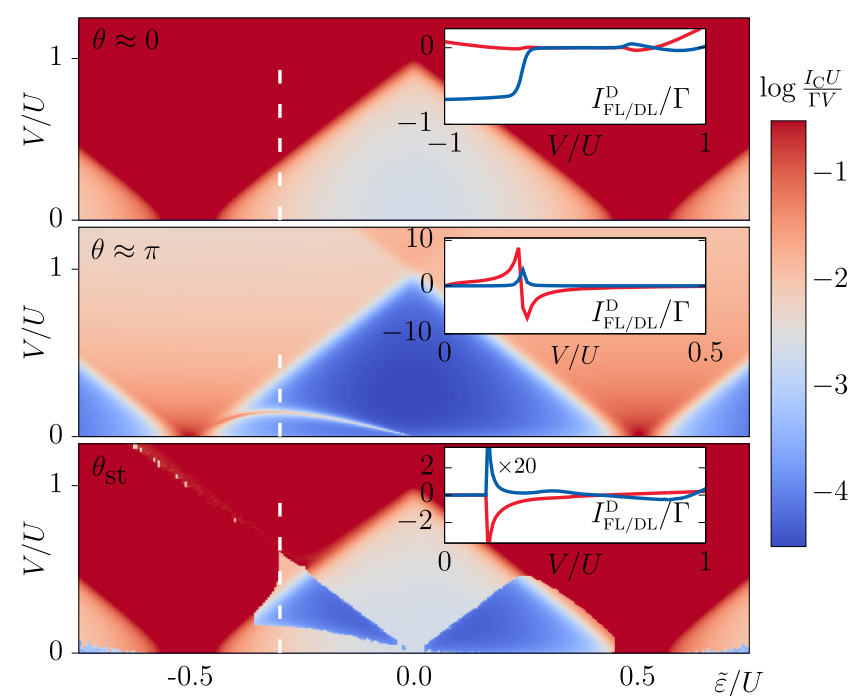

FIG. 3. Logarithmic representation of the charge current per applied voltage bias $\log \left(I_{\mathrm{C}} / V\right)$ in the nearly parallel $(\theta=0.01 \pi)$, nearly antiparallel $(\theta=0.99 \pi)$, and dynamic cases (from Fig. 2). All parameters are as in Fig. 2. The upper part of the color scale relevant for transport outside of the Coulomb blockade is omitted. Generically, the parallel alignment results in larger currents than the antiparallel alignment. Insets: fieldlike (red) and dampinglike (blue) spin torques along cuts at $\tilde{\varepsilon}=-0.3 U$, indicated by dashed white lines in the main figure.

The actual switching from antiparallel to parallel always occurs via a fast transition, while the inverse process is significantly influenced by temperature. The underlying reason for this is the absence of spin torques in the parallel limit (see insets in Fig. 4). Hence, thermal fluctuations first have to generate a sufficiently large initial opening angle for the fieldlike spin torque to take over and drive the actual switching. As this waiting time is usually much longer than the actual spin-torque driven switching time [23], this effect dominates. On the other hand, due to the stronger spin torques, the antiparallel alignment-as long as it is supported by the spin current-is highly robust against thermal fluctuations, counterintuitively, in particular, for $(\varepsilon, V)$ values close to the resonance where the transition from antiparallel to parallel alignment occurs. In contrast, going across the transition line in the $(\varepsilon, V)$-parameter regime will cause similar strong spin currents to easily switch the nanomagnets to a parallel alignment.

Ferromagnetic resonance.-We now turn to the spin torques shown in Fig. 4. These are accessible via ferromagnetic resonance (FMR) experiments. We assume that the corresponding external magnetic field for such a setup is small enough to not effectively lift the degeneracy of the quantum dot, i.e., $B_{\text {extern }} \ll \Gamma$. For a larger external magnetic field, the precessing quantum dot spin would be pinned, and the effects discussed here will be suppressed.

FMR measures the absorption of electromagnetic waves at a given frequency $\Omega$. The response of the $x$ component of the drain spin density to, for example, a linearly polarized

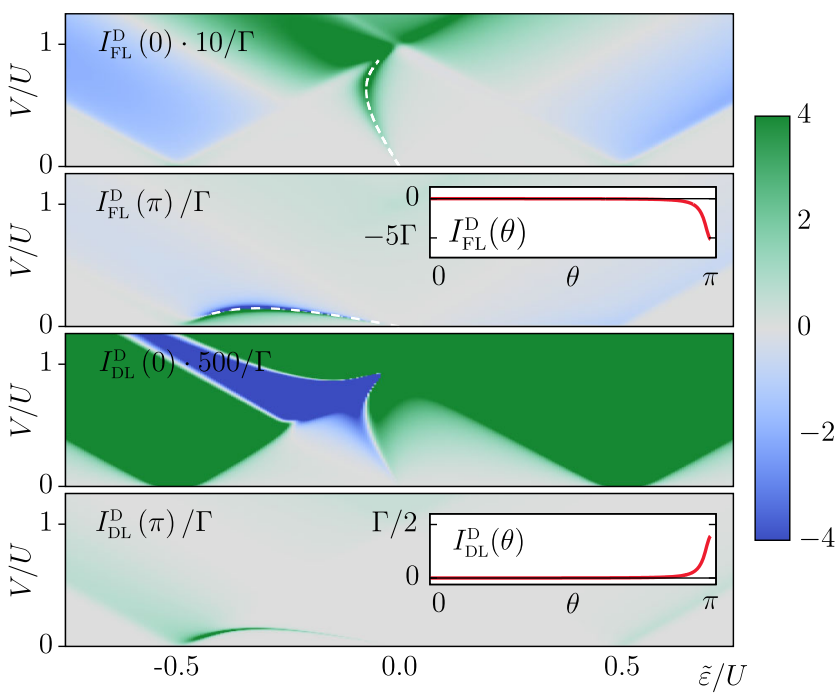

FIG. 4. Field- and dampinglike spin torques $I_{\mathrm{FL}}^{D}$ and $I_{\mathrm{DL}}^{D}$ for the parallel (respective upper) and antiparallel (respective lower) cases. All parameters are as in Fig. 2. The dashed lines correspond to the resonance conditions $\vec{B}_{\text {ind }} \cdot\left(\vec{n}_{D} \pm \vec{n}_{S}\right)=0$, with the upper and lower sign valid for $\theta \approx 0$ and $\theta \approx \pi$, respectively. The full phase diagrams are obtained from the symmetry and antisymmetry of $I_{\mathrm{FL}}^{D}$ and $I_{\mathrm{DL}}^{S}$ under $(\tilde{\varepsilon}, V) \rightarrow(-\tilde{\varepsilon},-V)$. Insets: Angular dependence of the respective spin torque for $\tilde{\varepsilon}=-U / 4, V=0.15 U$ close to the resonance (4).

driving field $\mathbf{h}(t)=h_{x} \cos (\Omega t) \hat{\mathbf{x}}$ is given by $n_{D, x}(t)=$ $\chi_{x x}^{\prime} h_{x} \cos (\Omega t)+\chi_{x x}^{\prime \prime} h_{x} \sin (\Omega t)$. Assuming $\alpha \ll 1$, the outof-phase susceptibility $\chi_{x x}^{\prime \prime}$ is, for example [23],

$\chi_{x x}^{\prime \prime}=S \Omega \frac{-2 S^{2} \Omega_{R}^{2} \alpha_{\mathrm{eff}}+\alpha\left[\left(I_{\mathrm{DL}}^{D}\right)^{2}+S^{2}\left(\Omega_{R}^{2}-\Omega^{2}\right)\right]}{\left[\left(I_{\mathrm{DL}}^{D}\right)^{2}+S^{2}\left(\Omega_{R}^{2}-\Omega^{2}\right)\right]^{2}+\left(2 S^{2} \alpha_{\mathrm{eff}} \Omega \Omega_{R}\right)^{2}}$,

where $\Omega_{R}=\left(\gamma H-I_{\mathrm{FL}}^{D}\right) / S$ and $\alpha_{\mathrm{eff}}=\alpha+I_{\mathrm{DL}}^{D} /\left(S \Omega_{R}\right)$. Hence, the fieldlike torque causes a shift of the resonance position, while the dampinglike torque alters the broadening $\sim \alpha_{\text {eff }}$.

As shown in Fig. 4, for an antiparallel configuration $\theta \approx \pi$, the field- and dampinglike torques are of the same order and both clearly exhibit a feature following the resonance condition (4). On the other hand, for the parallel configuration, the dampinglike spin torque inside the Coulomb blockade is heavily suppressed. Furthermore, instead of the resonance at (4), both the field- and dampinglike torque possess a new feature at $\vec{B}_{\text {ind }} \cdot\left(\vec{n}_{D}+\vec{n}_{S}\right)=0$. This resonance is the partner of (4) in the sense that, instead of maximizing, it minimizes the efficiency of the induced magnetic field to rotate the spin from the source to the drain direction or vice versa. It also appears in the cotunneling charge current through the quantum dot and yields a very weak conductance peak (invisible in Fig. 3). If the opening angles start to have a relevant deviation from the nearly parallel setup $\theta \gtrsim 0.1 \pi$, the resonance following (4) quickly begins to dominate. 
Controlling the spin torques.-Generally, the strong dependence of the field- and dampinglike spin torques on the tunable gate voltage $\varepsilon$ (via the on-site energy $\tilde{\varepsilon}$ ) and the applied voltage bias $V$ allows for experimental control of these normally fixed parameters. The insets of Fig. 3 show how the spin torques vary as a function of the bias voltage $V$, corresponding to cuts in Fig. 4. For example, in the fixed parallel case, one can switch on the dampinglike torque by leaving the Coulomb diamond. In contrast, the fixed antiparallel setup has significant features only inside the Coulomb blockade region where around the resonance at (4), the sign of the fieldlike torque can be flipped.

Conclusions.-We showed that quantum transport through a spin-degenerate quantum dot provides unique control over the spin torques acting on the attached nanomagnets, thus enabling the effective switching of the nanomagnets from a parallel to an antiparallel configuration and vice versa. At the same time, the cotunneling charge current through the system is a reliable readout tool for the magnetic configuration of the nanomagnets. Our finding relies on the spin degeneracy of the quantum dot level; adding further aspects to the model like additional quantum levels or phonon degrees of freedom is not expected to change our results qualitatively.

We would like to thank Gerrit Bauer, Michael Hell, Martin Leijnse, Yaroslav Tserkovnyak, and Maarten Wegewijs for useful comments and discussions. This work is part of the D-ITP consortium, a program of the Netherlands Organisation for Scientific Research (NWO) that is funded by the Dutch Ministry of Education, Culture, and Science $(\mathrm{OCW})$. This project has received funding from the European Research Council (ERC) under the European Union's Horizon 2020 research and innovation programme (Grant Agreement 725509-SPINBEYOND).

[1] M. N. Baibich, J. M. Broto, A. Fert, F. N. Van Dau, F. Petroff, P. Etienne, G. Creuzet, A. Friederich, and J. Chazelas, Phys. Rev. Lett. 61, 2472 (1988).

[2] R. E. Camley and J. Barnaś, Phys. Rev. Lett. 63, 664 (1989).

[3] P. M. Levy, S. Zhang, and A. Fert, Phys. Rev. Lett. 65, 1643 (1990).

[4] T. Valet and A. Fert, Phys. Rev. B 48, 7099 (1993).

[5] L. Berger, Phys. Rev. B 54, 9353 (1996).

[6] M. Tsoi, A. G. M. Jansen, J. Bass, W.-C. Chiang, M. Seck, V. Tsoi, and P. Wyder, Phys. Rev. Lett. 80, 4281 (1998).

[7] J. Slonczewski, J. Magn. Magn. Mater. 195, L261 (1999).

[8] Z. Diao, Z. Li, S. Wang, Y. Ding, A. Panchula, E. Chen, L.-C. Wang, and Y. Huai, J. Phys. Condens. Matter 19, 165209 (2007).

[9] D. Ralph and M. Stiles, J. Magn. Magn. Mater. 320, 1190 (2008).

[10] E. R. Evarts, L. Cao, D. S. Ricketts, N. D. Rizzo, J. A. Bain, and S. A. Majetich, Appl. Phys. Lett. 95, 132510 (2009).

[11] H.-A. Engel and D. Loss, Phys. Rev. B 65, 195321 (2002).
[12] J. König and J. Martinek, Phys. Rev. Lett. 90, 166602 (2003).

[13] J. Martinek, Y. Utsumi, H. Imamura, J. Barnaś, S. Maekawa, J. König, and G. Schön, Phys. Rev. Lett. 91, 127203 (2003).

[14] M. Braun, J. König, and J. Martinek, Phys. Rev. B 70, 195345 (2004).

[15] M. Braun, J. König, and J. Martinek, Europhys. Lett. 72, 294 (2005).

[16] M. Wilczyński, R. Świrkowicz, W. Rudziński, J. Barnaś, and V. Dugaev, J. Magn. Magn. Mater. 290-291, 209 (2005).

[17] R. Świrkowicz, M. Wilczyński, and J. Barnaś, J. Phys. Condens. Matter 20, 255219 (2008).

[18] C. A. Merchant and N. Marković, Phys. Rev. Lett. 100, 156601 (2008).

[19] M. Hell, B. Sothmann, M. Leijnse, M. R. Wegewijs, and J. König, Phys. Rev. B 91, 195404 (2015).

[20] J. Slonczewski, J. Magn. Magn. Mater. 159, L1 (1996).

[21] S. Rafique, J. R. Cullen, M. Wuttig, and J. Cui, J. Appl. Phys. 95, 6939 (2004).

[22] J. B. Restorff, M. Wun-Fogle, A. E. Clark, and K. B. Hathaway, IEEE Trans. Magn. 42, 3087 (2006).

[23] See Supplemental Material at http://link.aps.org/ supplemental/10.1103/PhysRevLett.120.017701 which also includes Refs. [24-28], for technical details and additional, supporting results.

[24] H. Schoeller and F. Reininghaus, Phys. Rev. B 80, 045117 (2009); 80, 209901(E) (2009).

[25] N. M. Gergs, C. B. M. Hörig, M. R. Wegewijs, and D. Schuricht, Phys. Rev. B 91, 201107(R) (2015).

[26] D. E. Parkes, L. R. Shelford, P. Wadley, V. Holý, M. Wang, A. T. Hindmarch, G. van der Laan, R. P. Campion, K. W. Edmonds, S. A. Cavill, and A. W. Rushforth, Sci. Rep. 3, 2220 (2013).

[27] P. Puczkarski, P. Gehring, C. S. Lau, J. Liu, A. Ardavan, J. H. Warner, G. A. D. Briggs, and J. A. Mol, Appl. Phys. Lett. 107, 133105 (2015).

[28] A. Barreiro, H. S. J. van der Zant, and L. M. K. Vandersypen, Nano Lett. 12, 6096 (2012).

[29] S. Ament, N. Rangarajan, and S. Rakheja, arXiv:1607.04596.

[30] J. Xiao, G. E. W. Bauer, and A. Brataas, Phys. Rev. B 77, 224419 (2008).

[31] R. B. Saptsov and M. R. Wegewijs, Phys. Rev. B 86, 235432 (2012)

[32] B. Muralidharan and M. Grifoni, Phys. Rev. B 88, 045402 (2013).

[33] S. Hoffman and Y. Tserkovnyak, Phys. Rev. B 91, 245427 (2015).

[34] M. Leijnse and M. R. Wegewijs, Phys. Rev. B 78, 235424 (2008).

[35] H. Schoeller, Eur. Phys. J. Spec. Top. 168, 179 (2009).

[36] M. Leijnse, M. R. Wegewijs, and M. H. Hettler, Phys. Rev. Lett. 103, 156803 (2009).

[37] S. Koller, M. Grifoni, M. Leijnse, and M. R. Wegewijs, Phys. Rev. B 82, 235307 (2010).

[38] R. B. Saptsov and M. R. Wegewijs, Phys. Rev. B 90, 045407 (2014).

[39] M. Brooks and G. Burkard, Phys. Rev. B 95, 245411 (2017). 